\title{
Biexciton state preparation in a quantum dot via adiabatic rapid passage: comparison between two control protocols and impact of phonon-induced dephasing
}

\author{
M. Glässl1 ${ }^{1}$. A. Barth ${ }^{1}, \mathrm{~K} . \mathrm{Gawarecki}^{2,3}, \mathrm{P} . \mathrm{Machnikowski}^{2}, \mathrm{M}$. D. \\ Croitoru $^{1}$, S. Lüker ${ }^{3}$, D. E. Reiter ${ }^{3}$, T. Kuhn ${ }^{3}$, and V. M. Axt ${ }^{1}$ \\ ${ }^{1}$ Institut für Theoretische Physik III, Universität Bayreuth, 95440 Bayreuth, Germany \\ ${ }^{2}$ Institute of Physics, Wroctaw University of Technology, 50-370 Wroctaw, Poland and \\ ${ }^{3}$ Institut für Festkörpertheorie, Universität Münster, 48149 Münster, Germany
}

(Dated: December 17, 2018)

\begin{abstract}
We investigate theoretically under which conditions a stable and high-fidelity preparation of the biexciton state in a quantum dot can be realized by means of adiabatic rapid passage in the presence of acoustic phonon coupling. Our analysis is based on a numerically complete real-time path integral approach and comprises different schemes of optical driving using frequency-swept (chirped) pulses. We show that depending on the size of the biexciton binding energy, resonant two-photon excitations or two-color schemes can be favorable. It is demonstrated that the carrier-phonon interaction strongly affects the efficiency of the protocols and that a robust preparation of the biexciton is restricted to positive chirps and low temperatures. A considerable increase of the biexciton yield can be achieved realizing temperatures below $4 \mathrm{~K}$.
\end{abstract}

\section{INTRODUCTION}

Realizing an on demand source of entangled photon pairs is crucial for many innovative applications in quantum information science $e^{\underline{1}}$ and quantum optics 2 . Related applications comprise quantum computation ${ }^{\underline{3}}$, quantum teleportation ${ }^{4}$, or quantum key distribution ${ }^{\frac{5}{2}}$ as well as tests of fundamental aspects of quantum mechanics. A very promising scheme to generate polarization entangled photon pairs is to use the radiative decay of the biexciton state (consisting of two electron-hole pairs) in

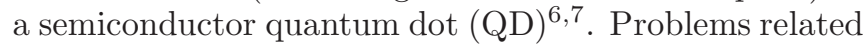
to the fine structure splitting of the intermediate exciton states have largely been overcome $e^{8,9}$ and recently, impressive experiments reported the realization of ultrabright sources by coupling a photonic molecule to a single QD 10 .

A necessary precondition for an efficient use of the biexciton decay cascade is a robust preparation of the biexciton state. In principle, an inversion of the QD from the ground to the biexciton state is possible by driving the system with a transform limited laser pulse of constant frequency, that is resonant to half of the ground state biexciton transition frequency 11,12 . The drawback of this Rabi-flopping scheme is that it requires a very detailed knowledge of the system parameters like the transition dipole moments as well as a very precise control of the field intensity in order to ensure the desired inversion. Recalling that these requirements are often only insufficiently fulfilled, it seems that a more practical way to realize a stable and high-quality preparation of the biexciton state is the use of optical driving schemes that rely on adiabatic rapid passage (ARP) and use frequencyswept (chirped) pulses. The basic idea underlying such schemes, that are stable with respect to intensity changes of the laser field, is to drive the system adiabatically along an eigenstate of the coupled light-matter Hamiltonian, whereby the character of the state changes during the pulse via a level anticrossing. Recently, experimen-

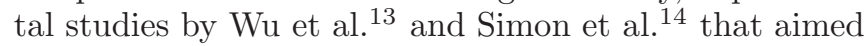
to prepare the single exciton state by means of ARP, have proven the applicability of an earlier proposed theoretical protocol ${ }^{15}$ and demonstrated a stable generation of the exciton state, that in the ARP-regime did only slightly depend on the applied pulse area. However, the efficiency reported in these experiments stayed below the ideal case and most recent theoretical calculations gave compelling evidence that this reduction can be attributed to the coupling of the QD to acoustic phonons 16 . It has been demonstrated that acoustic phonons lead to a drastic deterioration of the exciton generation for negative values of the chirp, while an efficient preparation can be achieved for positive chirps, where the phonon influence is restricted to phonon absorption processes that become unlikely at low temperatures $\underline{16}$ The reported drastic changes compared to the ideal scheme in the absence of the carrier-phonon interaction 15 emphasize the necessity to study the influence of the unavoidable coupling of carriers to acoustic phonons on other schemes relying on ARP in order to fully characterize their true potential.

In this paper, we discuss two different ARP based protocols aiming at an inversion of the QD to the biexciton state and give a comprehensive analysis of the phonon impact on these schemes. In the first protocol, the QD is driven by a single linearly polarized chirped pulse where the frequency at the pulse maximum is resonant to half of the ground state biexciton transition as first suggested by Hui and Liu ${ }^{17}$. In the second scheme, the QD is driven by two circularly polarized chirped pulses, that at the pulse maxima are resonant to the ground state exciton and to the exciton biexciton transition, respectively. For both protocols, the evolution of the system along an adiabatic branch is more complex than for two-level schemes. For example, in contrast to the two-level case, ARP is here sensitive to the sign of the chirp even without taking the carrier-phonon interaction into account. Therefore, it is a priori unclear to what extent the efficiency is affected 
by the coupling to acoustic phonons. It turns out, however, that similar to the results reported in Ref. 16 for the inversion to the exciton state, an efficient preparation of the biexciton is restricted to positive chirps and low temperatures. Quite significant differences between both investigated protocols arise for moderate and large values of the biexciton binding energy $\Delta$, as the pulse area threshold that has to be exceeded in order to ensure a purely adiabatic evolution rises strongly with $\Delta$ for the first scheme, whereas it is independent of $\Delta$ for the second protocol. Above the respective ARP-thresholds, the biexciton occupation depends slightly on the pulse area in a nonmonotonic way.

The paper is organized as follows. In Sec. [1] we outline the model and comment briefly on the real-time path integral method that is used for our numerical simulations. The latter are presented in Sec. III, where Sec. IIIA analyzes the phonon impact on the two-photon resonance scheme, while Sec. IIIB deals with the phonon influence on the two-color protocol and compares the efficiency of both schemes. Finally, Sec. IV concludes the paper.

\section{THEORY}

We consider an optically driven strongly confined GaAs QD coupled to a continuum of acoustic phonons. Our model Hamiltonian is defined by $H=H_{\text {dot,las }}^{\text {circlin }}+$ $H_{\text {dot,ph }}$, where the first term describes the electronic structure of the QD and the coupling to a laser of either circular or linear polarization, while the second term represents the carrier-phonon coupling. For excitations involving circularly $\sigma_{+}$as well as circularly $\sigma_{-}$polarized pulses, we account for four bright states: the ground state $|G\rangle$, the two single exciton states $\left|\sigma_{ \pm}\right\rangle$, and the biexciton state $|B\rangle$. In this basis, $H_{\text {dot,las }}^{\text {circ }}$ reads

$$
\begin{aligned}
H_{\mathrm{dot}, \text { las }}^{\text {circ }} & =\hbar \omega_{0}\left(\left|\sigma_{+}\right\rangle\left\langle\sigma_{+}|+| \sigma_{-}\right\rangle\left\langle\sigma_{-}\right|\right) \\
& +\left(2 \hbar \omega_{0}-\Delta\right)|B\rangle\langle B| \\
& +\frac{\hbar}{2}\left[f^{\sigma_{+}}(t)\left(\left|\sigma_{+}\right\rangle\langle G|+| B\rangle\left\langle\sigma_{-}\right|\right)+\text {h.c. }\right] \\
& +\frac{\hbar}{2}\left[f^{\sigma_{-}}(t)\left(\left|\sigma_{-}\right\rangle\langle G|+| B\rangle\left\langle\sigma_{+}\right|\right)+\text {h.c. }\right],
\end{aligned}
$$

where $f^{\sigma_{ \pm}}(t)=2 \mathbf{M} \cdot \mathbf{E}_{\sigma_{ \pm}}^{(+)}(t) / \hbar$ with $\mathbf{E}_{\sigma_{ \pm}}^{(+)}$being the positive frequency component of the $\sigma_{ \pm}$circularly polarized light field and $\mathbf{M}$ denoting the transition dipole element. $\hbar \omega_{0}$ defines the ground state exciton transition energy and $\Delta$ represents the biexciton binding energy. Electron-hole exchange interactions, that result in a direct coupling between the single exciton states are neglected, which is well justified for the picosecond time-scale considered here and a sufficiently small fine structure splitting (within $10 \mu \mathrm{eV}$ for the strongest chirps studied here). For linearly polarized excitations, it is advantageous to introduce the single exciton state $|X\rangle=\left(\left|\sigma_{+}\right\rangle+\left|\sigma_{-}\right\rangle\right) / \sqrt{2}$ which allows us to apply a model with only three levels $\stackrel{11,18}{=} H_{\text {dot,las }}^{\text {lin }}$ is then given by

$$
\begin{aligned}
H_{\text {dot,las }}^{\operatorname{lin}} & =\hbar \omega_{0}|X\rangle\left\langle X\left|+\left(2 \hbar \omega_{0}-\Delta\right)\right| B\right\rangle\langle B| \\
& +\left[\hbar f^{\operatorname{lin}}(t) / 2(|X\rangle\langle G|+| B\rangle\langle X|)+\text { h.c. }\right]
\end{aligned}
$$

with $f^{\operatorname{lin}}(t)=2 \mathbf{M} \cdot \mathbf{E}_{\text {lin }}^{(+)}(t) / \hbar$ and $\mathbf{E}_{\text {lin }}^{(+)}$denoting the positive frequency component of the linearly polarized electric field.

In this work, we will concentrate on optical driving by chirped Gaussian pulses. These frequency swept pulses can be obtained from transform limited Gaussian pulses of the form

$$
f_{0}(t)=\frac{A}{\sqrt{2 \pi \tau_{0}^{2}}} \exp \left(-\frac{t^{2}}{2 \tau_{0}^{2}}\right) \exp (-i \omega t),
$$

with the original pulse area $A$, the pulse duration $\tau_{0}$, and the central frequency $\omega$. Here, the polarization label used in Eqs. (11) and (2) has been suppressed for simplicity. Applying a Gaussian chirp filter ${ }^{19}$ with the chirp coefficient $\alpha$ transforms $f_{0}(t)$ into

$$
f(t)=\frac{A}{\sqrt{2 \pi \tau_{0} \tau}} \exp \left(-\frac{t^{2}}{2 \tau^{2}}\right) \exp \left(-i \omega t-i \frac{a t^{2}}{2}\right),
$$

where $\tau=\sqrt{\alpha^{2} / \tau_{0}^{2}+\tau_{0}^{2}}$ characterizes the chirped pulse length and $a=\alpha /\left(\alpha^{2}+\tau_{0}^{4}\right)$ is the frequency chirp rate. In the following, we will use pulses of different polarizations and central frequencies. Details will be given in Sec. III.

The carrier-phonon coupling is treated in terms of the independent Boson model ${ }^{20}$. Concentrating on the deformation potential coupling to longitudinal acoustic (LA) phonons, which has been shown to provide the dominant dephasing mechanism in GaAs QDs ${ }^{21}-23, H_{\text {dot,ph }}$ reads

$$
H_{\mathrm{dot}, \mathrm{ph}}=\sum_{\mathbf{q}} \hbar \omega_{\mathbf{q}} b_{\mathbf{q}}^{\dagger} b_{\mathbf{q}}+\sum_{\mathbf{q}, \nu} \hbar n_{\nu}\left(g_{\mathbf{q}} b_{\mathbf{q}}+g_{\mathbf{q}}^{*} b_{\mathbf{q}}^{\dagger}\right)|\nu\rangle\langle\nu| .
$$

The operator $b_{\mathbf{q}}^{\dagger}\left(b_{\mathbf{q}}\right)$ creates (annihilates) a LA phonon with wave vector $\mathbf{q}$ and energy $\hbar \omega_{\mathbf{q}}=\hbar c_{s} q$, where $c_{s}=5110 \mathrm{~m} / \mathrm{s}$ is the longitudinal sound velocity. $n_{\nu}$ denotes the number of excitons present in the state $|\nu\rangle$ and $g_{\mathbf{q}}=\left(g_{\mathbf{q}}^{\mathrm{e}}-g_{\mathbf{q}}^{\mathrm{h}}\right)$ represents the exciton-phonon coupling constant that is given as the difference between the electron-phonon and hole-phonon coupling constants

$$
g_{\mathbf{q}}^{\mathrm{e}(\mathrm{h})}=\Psi^{e(h)}(\mathbf{q}) D_{e(h)} \sqrt{\frac{q}{2 V \rho \hbar c_{s}}},
$$

where $V$ is the sample volume, $D_{e}=-14.6 \mathrm{eV}$ and $D_{h}=-4.8 \mathrm{eV}$ denote the deformation potential coupling constants of electrons and holes and $\rho=5370 \mathrm{~kg} / \mathrm{m}^{3}$ is the material density. $\Psi^{e(h)}$ represents the form factor of the carriers confined in the QD. For simplicity, we assume a spherical harmonic oscillator confinement, leading to

$$
\Psi^{e(h)}(\mathbf{q})=\exp \left(-q^{2} a_{e(h)}^{2} / 4\right) .
$$

We choose an electron confinement length of $a_{e}=3 \mathrm{~nm}$ and set $a_{h}=0.87 a_{e}$. 


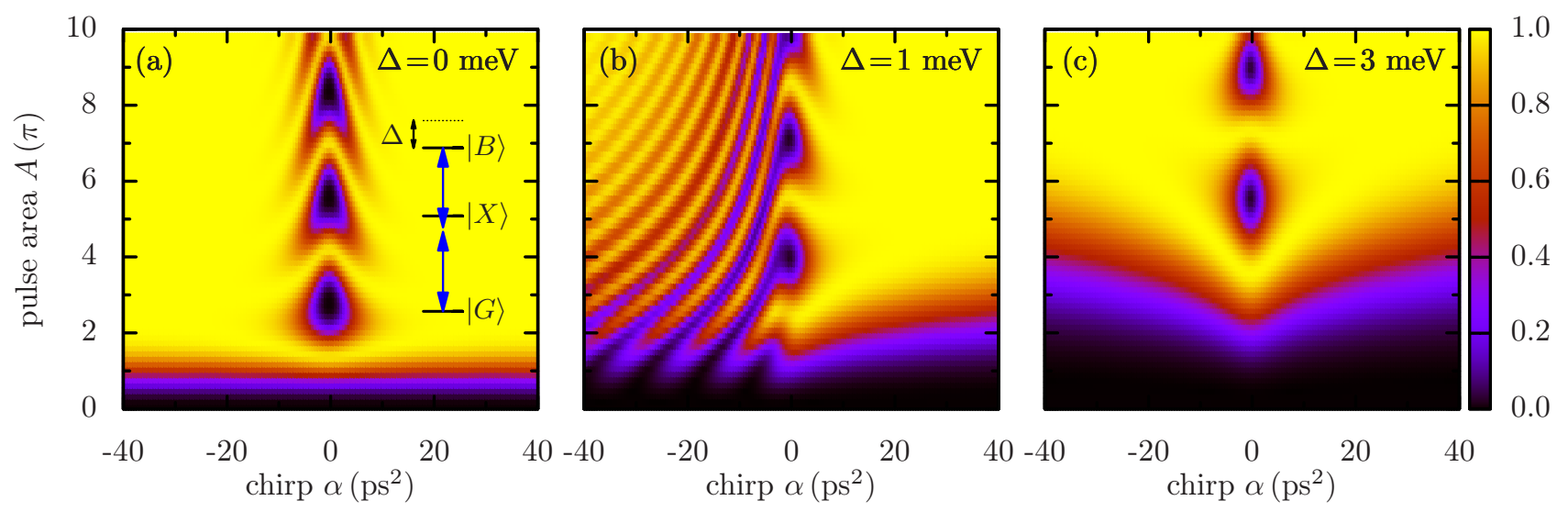

FIG. 1: (Color online) Final biexciton occupation after a linearly polarized and frequency-swept Gaussian pulse with $\tau_{0}=2$ ps as a function of the original pulse area $A$ and the chirp $\alpha$ for biexciton binding energies of (a) $\Delta=0$, (b) 1 , and (c) 3 meV in the absence of the carrier-phonon coupling. The central frequency (at zero chirp) is chosen such that the two-photon process is resonant to the ground state biexciton transition [cf. inset of panel (a)].

To analyze the combined carrier-phonon dynamics, we use a numerically exact real-time path-integral approach, that accounts fully for all non-Markovian effects and arbitrary multi-phonon processes. This allows us to study the phonon-influence on the driven dynamics at arbitrary temperatures and in parameter ranges, where approximate methods come to their limits 24 . A detailed description of the formalism is given in Ref. 25. Additional comments on the challenges that are faced within the path-integral approach when accounting for more than two electronic levels can be found in Ref. 18 .

\section{RESULTS}

In this section, we study how the presence of acoustic phonons affects the preparation of the biexciton state by means of adiabatic rapid passage. We shall analyze two protocols where in both cases we will apply chirps ranging from $\alpha=-40$ to $+40 \mathrm{ps}^{2}$ and choose an original pulse length of $\tau_{0}=2 \mathrm{ps}$ in order to use similar parameters as in recent experiments that reported on a robust quantum dot exciton generation via ARP13,14.

\section{A. Two-photon resonance ARP-scheme}

Let us first concentrate on a two-photon resonance ARP-scheme that uses one linearly polarized pulse. The central frequency of this pulse is chosen such that for zero chirp the two-photon process is resonant to the ground state biexciton transition, i.e., $\omega=\omega_{0}-\Delta /(2 \hbar)$, as schematically sketched in the inset of Fig. 1)(a).

To provide a reference for the phonon influence on the efficiency of this protocol, Fig. 1 shows the final biexciton occupation after the pulse as a function of the original pulse area $A$ and the chirp coefficient $\alpha$ for different values of the biexciton binding energy $\Delta$ neglecting the exciton-phonon coupling. For zero chirp, the biexciton occupation depends sensitively on the pulse intensity and performs Rabi rotations between the ground and the biexciton state with a Rabi period that strongly depends on the biexciton binding energy $y^{11}$. In contrast, for large enough positive chirps, an ideal and robust biexciton preparation, which is insensitive to small variations of the pulse area, can be achieved provided that the applied pulse area exceeds the ARP-threshold. The latter rises significantly with increasing $\Delta$ and shows for finite $\Delta$ also a considerable dependence on the strength of the chirp. Interestingly, the situation is different for negative chirps: for $\alpha<0$, a stable biexciton preparation can be only achieved for almost vanishing or large enough biexciton binding energies [cf. Figs. 1(a) and (c)], but fails for moderate values of $\Delta$, as it can be exemplarily seen from Fig. 1(b) for $\Delta=1 \mathrm{meV}$, where for $\alpha<0$ a stripe-like pattern forms. This dependence of the dynamics on the sign of the chirp contrasts to the widely studied ARPprotocols for the generation of the exciton state ${ }^{13}-16$ and has to be taken into account also in situations where one expects the influence of phonons to be negligible.

The results shown in Fig. 1 as well as the phonon impact that will be studied below can be most easily understood by analyzing the system evolution in the dressed state picture, i.e., by considering the instantaneous eigenstates and eigenenergies that are obtained by diagonalizing the combined light-matter Hamiltonian $H_{\text {dot,las. The }}^{\text {lin }}$ corresponding eigenenergies are plotted in Fig. 2 as a function of time for $\alpha=40 \mathrm{ps}^{2}$ and different values of the original pulse area $A$ as well as of the biexciton binding energy $\Delta$ together with the corresponding pulse envelope functions (shaded areas). Long before and long after the pulse maximum, it is possible to identify the instantaneous eigenstates with the electronic ground state $|G\rangle$, the single exciton state $|X\rangle$, and the biexciton state $|B\rangle$, as it is indicated in Fig. 2. During the pulse, the character of the eigenstates changes. For example, the lowest 

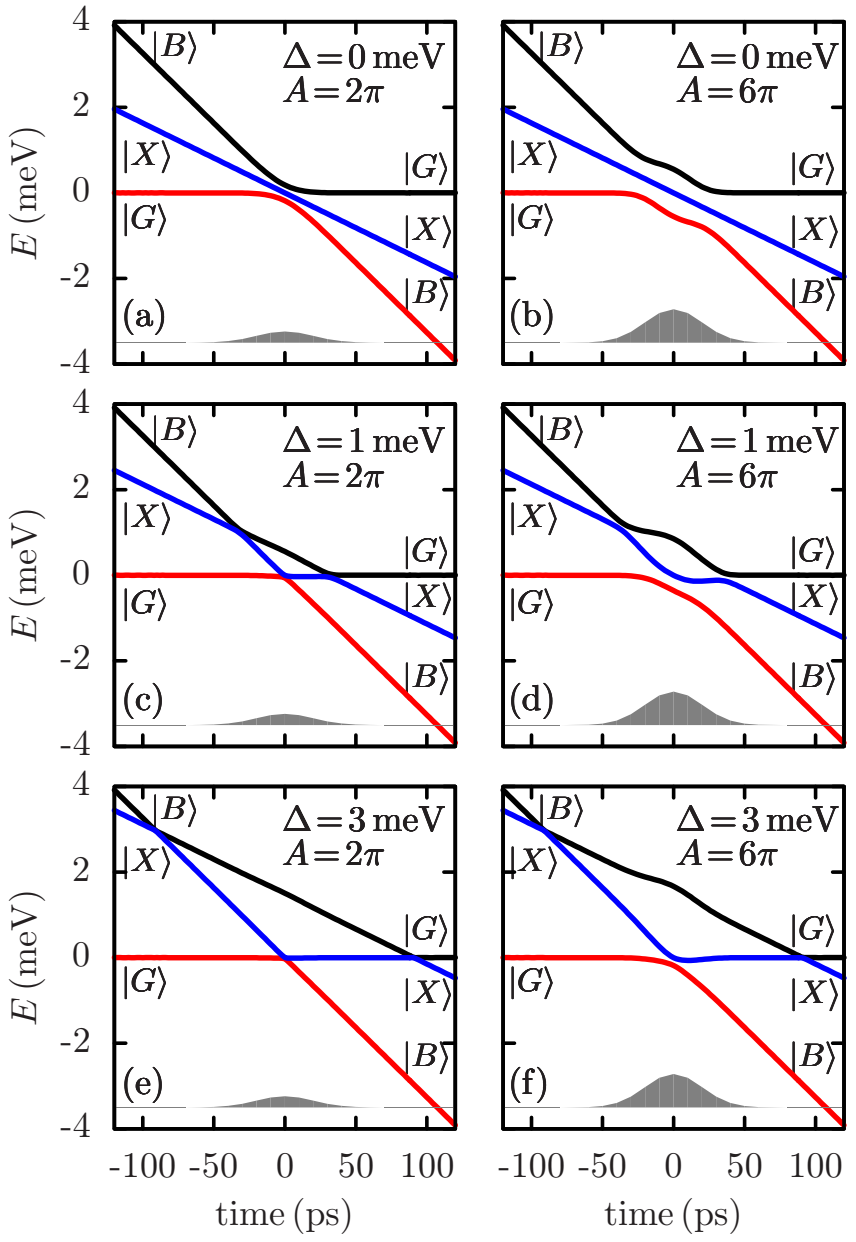

FIG. 2: (Color online) Adiabatic spectral branches of the laser driven $\mathrm{QD}$ system for the two-photon resonance ARPscheme for $\tau_{0}=2 \mathrm{ps}, \alpha=40 \mathrm{ps}^{2}$ and pulse areas and biexciton binding energies as indicated. The evolution for $\alpha=-40 \mathrm{ps}^{2}$ can be read off the plots by following the branches from the right to the left. The gray shaded areas represent the pulse envelope functions, that have been shifted downwards for clarity.

branch, that can initially be identified with $|G\rangle$, eventually transforms into $|B\rangle$. It is precisely this transformation that allows a stable biexciton preparation within the two-photon resonance ARP-scheme for positive values of $\alpha$ when the condition for adiabatic passage is fulfilled, i.e., when the change in the frequency and the change in the amplitude of the pulse are slow compared to the Rabi frequency ${ }^{26}$. In this case the system evolves along a single branch and passes all anticrossings. Obviously, both conditions are better fulfilled for higher pulse areas (corresponding to higher Rabi frequencies), which can be nicely seen from Fig. 2 by comparing the left and the right column, that show the branches for $A=2 \pi$ and $A=6 \pi$, respectively: the splitting of the anticrossings considerably increases with rising $A$. By comparing the different rows of Fig. 2 it can also be seen that for a given pulse area, the anticrossings become narrower for larger biexciton binding energies, explaining the rising threshold for ARP with increasing $\Delta$ as shown in Fig. 11. While for $A=2 \pi$ and $\Delta=0 \mathrm{meV}$ [cf. Fig. 2(a)] the splitting of the central anticrossing at $t=0$ is large enough to ensure an adiabatic passage resulting in the preparation of the biexciton state [cf. Fig. 1(a)], the situation is different for larger $\Delta$. When the anticrossing becomes too narrow [cf. Fig. 2(c) and (e)], the chirp rate is no longer small compared to the spacing between the branches, the condition for ARP is violated and the system does not evolve along a single branch.

Considering negative chirps, the situation is slightly more complex. For $\alpha=-40 \mathrm{ps}^{2}$ the system evolution along the instantaneous eigenstates can easily be derived from Fig. 2 by reading the plots from the right to the left (i.e., by replacing the time $t$ by $-t$ ). Obviously, a transformation from $|G\rangle$ into $|B\rangle$ is here possible by following the uppermost branch. In Figs. 2(a) and (b) for $\Delta=0$, one passes two anticrossings along the upper branch and the system evolves completely adiabatically. For $\Delta=3 \mathrm{meV}$, both anticrossings are very narrow and the system jumps two times from one branch to another branch instead of following the anticrossings. Both crossings together result again in the preparation of the biexciton, as it can also be seen in Fig. 1(c). For $\Delta=1 \mathrm{meV}$, the anticrossings are narrow, but still considerably wider than for larger biexciton binding energies. In consequence, the system does not evolve completely adiabatically (via pure anticrossings) nor purely non-adiabatically (via pure crossings) and a Rabi-like behavior is realized instead of a stable and robust preparation of the biexciton giving rise to the stripe-like pattern as seen in Fig. 1(b) for $\alpha<0$. The details of this pattern can be explained as follows: The fringes which develop from the occupation maxima or minima of the usual Rabi rotations that are realized at $\alpha=0$ decline for rising chirps because of two reasons: first, with increasing chirp the effective pulse area exceeds more and more the original pulse area [as it can be seen by comparing Eqs. (3) and (4)] and second, the Rabi period decreases with increasing detuning 27 . This leads to an increased number of occupation maxima or minima compared to $\alpha=0$. The attenuation of the stripe pattern in the range of high pulse areas and large chirps is due to an increasing splitting between the adiabatic branches that leads to a more and more adiabatic evolution.

Next, we shall include the carrier-phonon interaction. Shown in Figs. 3(a), (b), and (c) is the final biexciton occupation after the pulse as a function of the original pulse area $\mathrm{A}$ and the chirp coefficient $\alpha$ at a temperature of $T=4 \mathrm{~K}$ for the same biexciton binding energies as considered in Fig. 1]. Compared to the ideal evolution in the absence of acoustic phonons there are similarities as well as obvious differences. As in the phonon-free case the ARP-threshold in the pulse-area rises with increasing biexciton binding energies and for $\Delta=1 \mathrm{meV}$ and negative chirps, the stripe-like pattern discussed above is still visible. However, it is clearly seen that within the full 

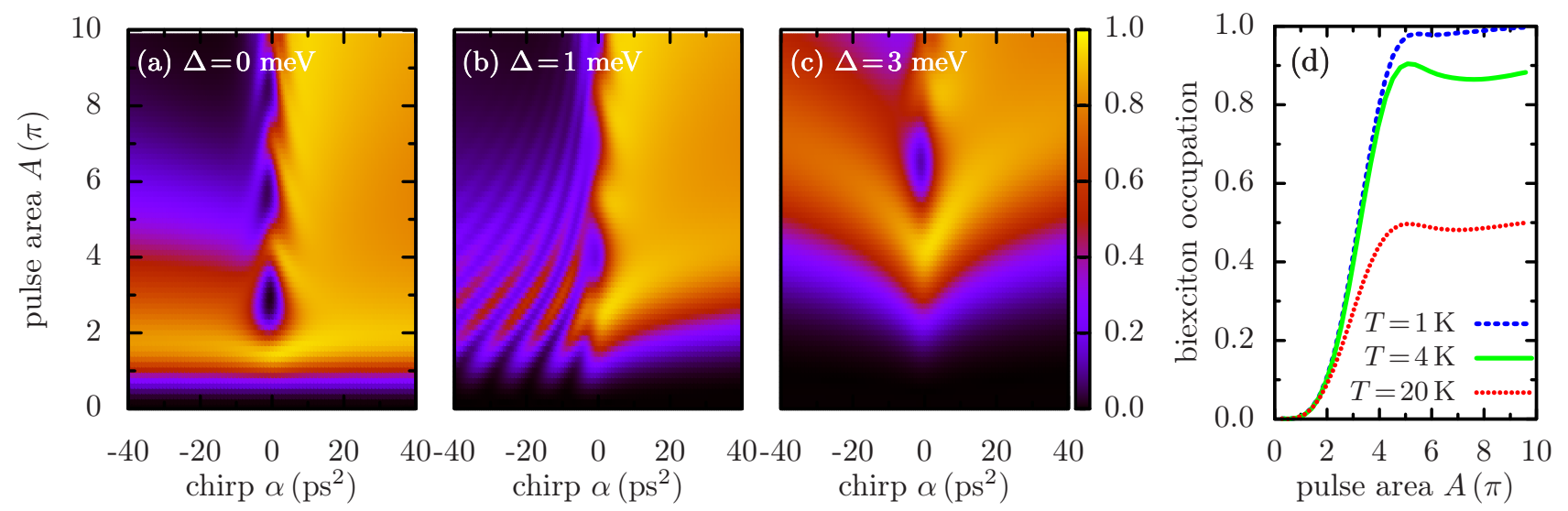

FIG. 3: (Color online) Final biexciton occupation after a linearly polarized and frequency-swept Gaussian pulse with $\tau_{0}=2$ ps within the two-photon resonance ARP-scheme. In (a)-(c) the occupation of the biexciton is shown as a function of the original pulse area $A$ and the chirp $\alpha$ for biexciton binding energies of (a) $\Delta=0$, (b) 1 , and (c) $3 \mathrm{meV}$ at $T=4 \mathrm{~K}$. In (d) the final occupation is plotted as a function of the original pulse area $A$ for $\Delta=2 \mathrm{meV}$ and a chirp of $\alpha=20 \mathrm{ps}^{2}$ at different temperatures.

model, the efficiency of the protocol does now strongly depend on the sign of the chirp, regardless of the size of the biexciton binding energy. While for positive chirps a rather stable biexciton generation is reached, negative chirps result in general in very low occupations of the biexciton state, in particular at high pulse areas. To understand this difference, one should recall that for a positive chirp, the adiabatic evolution follows the lowest adiabatic branch (cf. Fig. 22). Phonon mediated transitions to one of the other two branches, that spoil the purely adiabatic evolution and hamper the preparation of the biexciton state, are for $\alpha>0$ thus only possible via phonon absorption processes. At $4 \mathrm{~K}$ the latter are rather weak and hence, there is a perceivable but small deterioration of the protocol efficiency compared to the phonon-free case. For negative chirps, in contrast, the adiabatic evolution of the system follows the uppermost branch and transitions to one of the lower branches are possible by phonon emission processes, which at low temperatures dominate. In consequence, the population of the upper branch is drastically reduced in the course of time and an efficient preparation of the biexciton via ARP is no longer possible.

The temperature-dependence of the efficiency of the two-photon ARP scheme is illustrated in Fig. 3(d), where the final biexciton occupation is shown as a function of $A$ for a chirped pulse with $\alpha=20 \mathrm{ps}^{2}$, a biexciton binding energy of $\Delta=2 \mathrm{meV}$ and three different temperatures of $T=1,4$ and $20 \mathrm{~K}$. While for $4 \mathrm{~K}$ and pulse areas above the ARP-threshold occupations of about $90 \%$ are reached, the situation drastically deteriorates at elevated temperatures, where phonon absorption processes gain in importance due to a higher number of thermal phonons. Already at $20 \mathrm{~K}$, the biexciton generation turns out to be very inefficient with typical biexciton occupations staying below 0.5 . On the contrary, it is interesting to note that for temperatures well below $4 \mathrm{~K}$, an almost ideal biexci- ton preparation can be realized as exemplarily shown for $T=1 \mathrm{~K}$, where in the adiabatic regime a high-fidelity preparation of the biexciton with occupations higher than 0.98 is achieved. This remarkably strong difference between 1 and $4 \mathrm{~K}$ contrasts to wide-spread expectations that for low temperatures the impact of phonons is not very sensitive to a variation of few Kelvins and emphasizes the importance of realizing low temperatures.

A closer look on the curves plotted in Fig. 3(d) reveals further, that for pulse intensities above the threshold, the biexciton occupation depends nonmonotonically on the pulse area: an initial decrease is followed by a slight increase. This nonmonotonic behavior in the ARP regime corresponds to the reappearance of Rabi rotations in the usual Rabi regime ${ }^{28,29}$ and can be traced back to the resonance character of the carrier-phonon coupling 30 . The slow increase of the signal at high pulse areas as shown in Fig. 3(d) is mainly due to the Gaussian pulse envelope, which in the Rabi regime is known to lead to a rather weak reappearance because of an incomplete dynamical decoupling between lattice vibrations and electronic dynamics $\underline{29}$

\section{B. Two-color pulse ARP-scheme}

Let us next turn to an alternative ARP-scheme. The QD is now driven by two simultaneously applied frequency swept pulses of equal chirp, where the first pulse is circularly $\sigma_{+}$polarized and at the pulse maximum resonant to the ground state exciton transition, i.e., $\omega_{1}=\omega_{0}$, while the second pulse is circularly $\sigma_{-}$polarized and at its maximum in resonance to the exciton biexciton transition, i.e., $\omega_{2}=\omega_{0}-\Delta / \hbar$. A schematic sketch of this two-color protocol is drawn as an inset in Fig. 廿4(a). Neglecting the exciton-phonon coupling, also the two-color scheme allows for an ideal and stable preparation of the 

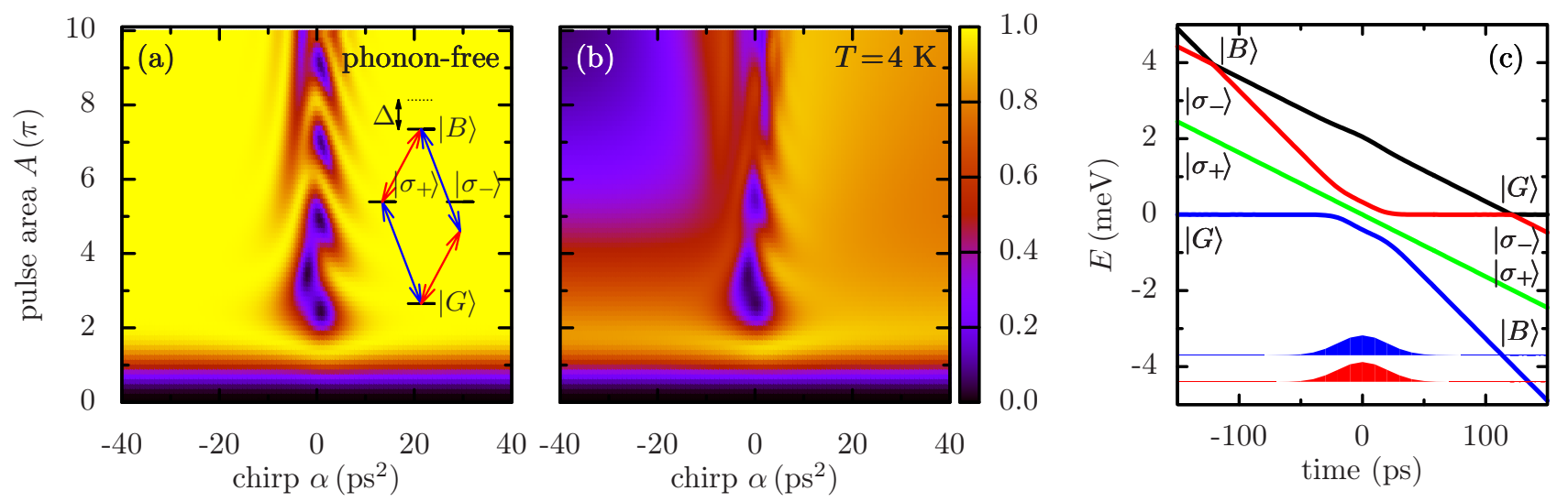

FIG. 4: (Color online) Results for the two-color pulse ARP-scheme that is schematically sketched in the inset of panel (a). In (a) and (b) the final biexciton occupation is shown as a function of the original pulse area $A$ of each circularly polarized pulse and the chirp $\alpha$ for $\tau_{0}=2 \mathrm{ps}$ and a biexciton binding energy of $\Delta=2 \mathrm{meV}$ in the phonon-free case (a) and in the full model at $T=4 \mathrm{~K}(\mathrm{~b})$. Shown in (c) is the evolution of the adiabatic spectral branches for $\tau_{0}=2 \mathrm{ps}, A=4 \pi, \alpha=40 \mathrm{ps}^{2}$ and $\Delta=2 \mathrm{meV}$. The evolution for $\alpha=-40 \mathrm{ps}^{2}$ can be read off the plot by following the branches from the right to the left. The shaded areas represent the envelope functions of both pulses, that have been shifted downwards for clarity.

biexciton. As an example, Fig. 4(a) shows the final biexciton occupation as a function of $A$ and $\alpha$ for $\Delta=2 \mathrm{meV}$, where $A$ now denotes the original pulse area of each circularly polarized pulse. We checked that even considerable deviations between both pulses with regard to the pulse intensity or the frequency sweep do not reduce the efficiency of the scheme provided that both components fulfill the condition for ARP. Also a finite delay between both pulses has no significant effect on the efficiency as long as the pulse that drives the ground state exciton transition precedes the pulse driving the exciton biexciton transition. Thus, the protocol is robust with respect to these changes and therefore, it is justified to concentrate on the case of equal pulse parameters and zero delay for reasons of simplicity.

An analysis of the adiabatic spectral branches for the two-color protocol shares many similarities with that given in Sec. IIIA for the two-photon resonance scheme and shall therefore not be presented at length. For positive chirps, the ground state transforms into the biexciton state via the lowest branch, whereas for negative chirps, the same transformation can be achieved by following the uppermost branch [cf. Fig. 4(c)]. Therefore, in the presence of the carrier-phonon coupling, a stable preparation is again only possible for positive chirps, as illustrated in Fig. 4(b) for $T=4 \mathrm{~K}$. A further similarity to the first protocol is that the two-color scheme is even in the phonon-free case sensitive to the sign of the chirp and that for negative chirps and small biexciton binding energies, a Rabi-like behavior is realized due to a neither purely adiabatic nor purely non-adiabatic evolution. The resulting pattern seen in the occupation when recorded as a function of $A$ and $\alpha$ (not shown) resembles that discussed in Fig. 1(b). Here, these patterns are most pronounced for $\Delta \sim 0.5 \mathrm{meV}$, which is half of the value of $\Delta \sim 1 \mathrm{meV}$, where the Rabi-like behavior is strongest in the two-photon resonance scheme. For these values of
$\Delta$, the detuning of the respective off-resonant levels is identical for both protocols.

Despite these similarities, one can also note significant and interesting differences between both considered protocols. Unlike for the two-photon resonance scheme, for the two-color protocol the pulse area threshold that must be exceeded in order to ensure an adiabatic dynamics is independent of the biexciton binding energy, as the transition to the biexciton does never involve excitation paths via strongly detuned intermediate levels. Independent of the chirp, the threshold is roughly given by $A=2 \pi$. Therefore, the two-color protocol is particularly advantageous for QDs with large biexciton binding energies. For example, for a high but still realistic value of $\Delta=5 \mathrm{meV}$

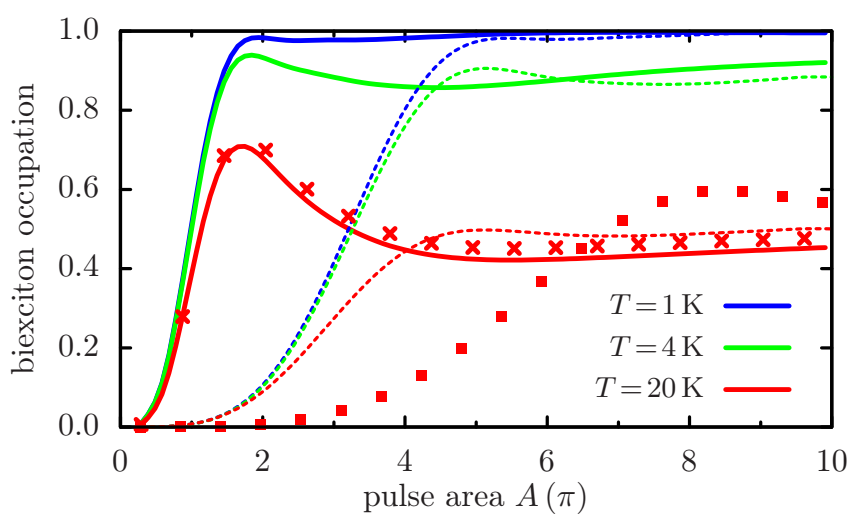

FIG. 5: (Color online) Final biexciton occupation after the two-color pulse ARP-scheme (solid lines) and the two-photon resonance ARP-scheme (dashed lines) as a function of the original pulse area $A$ for $\Delta=2 \mathrm{meV}$ and $\alpha=20 \mathrm{ps}^{2}$ at different temperatures. Crosses and squares represent results for $\Delta=5 \mathrm{meV}, \alpha=20 \mathrm{ps}^{2}$ and $T=20 \mathrm{~K}$ for the two-color pulse ARP-protocol and the two-photon resonance ARP-protocol, respectively. 
and a chirp of $\alpha=20 \mathrm{ps}^{2}$, the two-photon resonance scheme would require pulse areas above $8 \pi$ to reach the adiabatic limit and thus sixteen times as intense pulses as within the two-color protocol. This is exemplarily shown in Fig. 5 for $T=20 \mathrm{~K}$.

Besides the threshold characteristics, the detailed temperature and pulse area dependencies in the respective ARP-regime differ. This is shown in Fig. 5] where the biexciton occupation is plotted for both protocols as a function of the pulse area for a chirp of $\alpha=20 \mathrm{ps}^{2}$ and at temperatures of 1,4 , and $20 \mathrm{~K}$. Solid lines represent the results within the two-color scheme and dashed lines the results of the two-photon resonance protocol that have already been discussed in Fig. 3(d). While for $T=20 \mathrm{~K}$ the two-color scheme is for pulse areas around $2 \pi$ much more efficient than the two-photon resonance protocol, the situation is reversed at high pulse areas, where the efficiency is worse for the two-color scheme. For low temperatures, the two-color protocol performs slightly better, in particular at high pulse areas, where for $T=1 \mathrm{~K}$ an almost perfect inversion to the biexciton state is achieved. Common to the two-photon resonance scheme is the pronounced difference in the protocol efficiency between $T=1$ and $4 \mathrm{~K}$ as well as the nonmonotonic pulse area dependence. However, for the two-color scheme, the latter is slightly stronger and shows minima at smaller pulse areas. This finding is in line with recent calculations for transform limited pulses, that have revealed that the reappearance of Rabi rotations sets in at smaller pulse areas, when the polarization of the excitation is changed from linear to circular 31

\section{CONCLUSIONS}

We have analyzed the impact of acoustic phonons on two protocols aiming at a robust preparation of the biexciton state in a semiconductor QD. In the absence of the carrier-phonon interaction either driving the system with a single linearly polarized chirped pulse tuned at its maximum to the two-photon resonance of the ground state biexciton transition or using a two-color scheme where two circularly polarized chirped pulses are at their maxima in resonance with the ground state exciton and the exciton biexciton transition, respectively, enables a perfect preparation of the biexciton state provided that the conditions for adiabatic rapid passage are fulfilled. The most remarkable difference between both schemes, which is seen already without phonons, is the finding that the threshold for ARP rises drastically for the first proto- col with rising biexciton binding energies, while in the second protocol there is essentially no such dependence on the threshold. Different from related investigations of the preparation of the exciton state we here find a strong asymmetry with respect to the sign of the frequency sweep already in the idealized phonon-free case. In particular, we observe for finite negative chirps and intermediate biexciton binding energies a Rabi-like behavior, indicating a regime where the system neither evolves fully adiabatically along the adiabatic spectral branches nor does it switch between different branches without interference as it would be the case in the extreme nonadiabatic limit.

Accounting for phonons in general reduces the fidelity of both protocols and introduces an additional asymmetry with respect to the sign of the chirp that, as also known from earlier studies of the exciton preparation, results from the fact that for positive chirp the phonon emission is suppressed. A robust biexciton preparation is thus restricted to positive chirps and low temperatures. It is interesting to note that already at $4 \mathrm{~K}$ the efficiency is reduced to about $90 \%$ while at $1 \mathrm{~K}$ an almost perfect biexciton preparation is possible in spite of the phonons.

Taking the efficiency of the biexciton preparation as the only criterion it turns out that the two-color scheme performs slightly better for low temperatures. Moreover, for all temperatures the threshold is lower. Nevertheless, evaluating both schemes for practical usability these advantages should be weighted against some obvious drawbacks: the need of two pulses with different colors and (when thinking of using the biexciton decay cascade) the problem of laser stray light at the detection wavelength. The latter problem can be overcome by using up-conversion techniques 32 or non-resonant emittercavity coupling 33 . Thus, by paying the price of an increased effort one can indeed benefit from the better performance of the two color scheme. We expect that our analysis shall be a helpful guidance for making an adequate decision between performance and effort in upcoming experiments.

\section{ACKNOWLEDGMENTS}

M. G. is grateful for financial support from the Studienstiftung des Deutschen Volkes. M. D. C. is supported by the Alexander von Humboldt Foundation. P. M. and T. K. gratefully acknowledge financial support in the framework of a Research Group Linkage Project of the Alexander von Humboldt Foundation.
* martin.glaessl@uni-bayreuth.de

1 D. Bouwmeester, A. K. Ekert, and A. Zeilinger, The Physics of Quantum Information (Springer, Berlin, 2000).

${ }^{2}$ S. Haroche and J. Raimond, Exploring the quantum (Ox- ford University Press, Oxford, 2006), 1st ed.

3 M. A. Nielsen and I. Chuang, Quantum Computation and Quantum Information (Cambridge University Press, England, 2000). 
${ }^{4}$ D. Bouwmeester, P. J., K. Mattle, M. Eibl, H. Weinfurter, and A. Zeilinger, Nature 390, 575 (1997).

${ }^{5}$ N. Gisin, G. Ribordy, W. Tittel, and H. Zbinden, RMP 74, 145 (2002).

${ }^{6}$ E. Moreau, I. Robert, L. Manin, V. Thierry-Mieg, J. M. Gérard, and I. Abram, Phys. Rev. Lett. 87, 183601 (2001).

7 R. Stevenson, R. J. Young, P. Atkinson, K. Cooper, D. A. Ritchie, and A. J. Shields, Nature 439, 179 (2006).

8 N. Akopian, N. H. Lindner, E. Poem, Y. Berlatzky, J. Avron, D. Gershoni, B. D. Gerardot, and P. M. Petroff, Entangled Photon Pairs from Semiconductor Quantum Dots, vol. 96 (2006).

9 R. Trotta, E. Zallo, C. Ortix, P. Atkinson, J. D. Plumhof, J. van den Brink, A. Rastelli, and O. G. Schmidt, Phys. Rev. Lett. 109, 147401 (2012).

10 A. Dousse, J. Suffczyński, A. Beveratos, O. Krebs, A. Lemaître, I. Sagnes, J. Bloch, P. Voisin, and P. Senellart, Nature 466, 217 (2010).

11 P. Machnikowski, Phys. Rev. B 78, 195320 (2008).

12 S. Stufler, P. Machnikowski, P. Ester, M. Bichler, V. M. Axt, T. Kuhn, and A. Zrenner, Phys. Rev. B 73, 125304 (2006).

13 Y. Wu, I. M. Piper, M. Ediger, P. Brereton, E. R. Schmidgall, P. R. Eastham, M. Hugues, M. Hopkinson, and R. T. Phillips, Phys. Rev. Lett. 106, 067401 (2011).

14 C. M. Simon, T. Belhadj, B. Chatel, T. Amand, P. Renucci, A. Lemaitre, O. Krebs, P. A. Dalgarno, R. J. Warburton, X. Marie, et al., Phys. Rev. Lett. 106, 166801 (2011).

15 E. R. Schmidgall, P. R. Eastham, and R. T. Phillips, Phys. Rev. B 81, 195306 (2010).

16 S. Lüker, K. Gawarecki, D. E. Reiter, A. Grodecka-Grad, V. M. Axt, P. Machnikowski, and T. Kuhn, Phys. Rev. B 85, 121302 (2012). K. Gawarecki et al., arXiv:1209.2675.

17 H. Y. Hui and R. B. Liu, Phys. Rev. B 78, 155315 (2008).
18 M. Glässl, M. D. Croitoru, A. Vagov, V. M. Axt, and T. Kuhn, Phys. Rev. B 85, 195306 (2012).

19 B. Saleh and M. Teich, Fundamentals of Photonics, Wiley Series in Pure and Applied Optics (Wiley Interscience, New York, 2007).

20 G. D. Mahan, Many-Particle Physics (Plenum Press, New York, 1990), 2nd ed.

21 A. Vagov, V. M. Axt, T. Kuhn, W. Langbein, P. Borri, and U. Woggon, Phys. Rev. B 70, 201305(R) (2004).

22 A. J. Ramsay, A. V. Gopal, E. M. Gauger, A. Nazir, B. W. Lovett, A. M. Fox, and M. S. Skolnick, Phys. Rev. Lett. 104, 017402 (2010).

23 B. Krummheuer, V. M. Axt, and T. Kuhn, Phys. Rev. B 65, 195313 (2002).

24 M. Glässl, A. Vagov, S. Lüker, D. E. Reiter, M. D. Croitoru, P. Machnikowski, V. M. Axt, and T. Kuhn, Phys. Rev. B 84, 195311 (2011).

25 A. Vagov, M. D. Croitoru, M. Glässl, V. M. Axt, and T. Kuhn, Phys. Rev. B 83, 094303 (2011).

26 V. S. Malinovsky and J. L. Krause, Eur. Phys. J. D 14, 147 (2001).

27 L. Allen and J. H. Eberly, Optical Resonance and TwoLevel Atoms (John Wiley and Sons, New York, 1975).

28 A. Vagov, M. D. Croitoru, V. M. Axt, T. Kuhn, and F. M. Peeters, Phys. Rev. Lett. 98, 227403 (2007).

29 M. Glässl, M. D. Croitoru, A. Vagov, V. M. Axt, and T. Kuhn, Phys. Rev. B 84, 125304 (2011).

30 P. Machnikowski and L. Jacak, Phys. Rev. B 69, 193302 (2004).

31 M. Glässl and V. M. Axt, unpublished.

32 M. Paillard, X. Marie, P. Renucci, T. Amand, A. Jbeli, and J. M. Gérard, Phys. Rev. Lett. 86, 1634 (2001).

33 S. Ates, S. M. Ulrich, A. Ulhaq, S. Reitzenstein, A. Löffler, S. Höfling, A. Forchel, and P. Michler, Nature Photonics 3, 724 (2009). 